\title{
Uso de Modelos Lineares Mistos na análise do efeito de atividade física no nível de glicemia de mulheres na menopausa
}

\author{
Nair Cristina Margarido Brondino' \\ Departamento de Matemática, Unesp, Bauru, SP \\ Eduardo Federighi Baisi Chagas ${ }^{2}$ \\ Universidade de Marília (UNIMAR), \\ Laboratório de Avaliação e Prescrição de Atividade Física (LAPE); \\ Henrique Luiz Monteiro ${ }^{3}$ \\ Laboratório de Avaliação e Prescrição de Atividade Física (LAPE), \\ Departamento de Educação Física, Unesp, Bauru, SP
}

\begin{abstract}
Resumo. Em estudos que envolvem medidas repetidas, cada unidade experimental é observada pelo menos duas vezes e, portanto, espera-se uma dependência entre as respostas observadas. Grande parte do esforço empregado na análise deste tipo de dado está relacionada com a modelagem da estrutura de correlações intra-indivíduos. Dentro desse cenário, o Modelo Linear Misto, técnica empregada neste trabalho, permite modelar essa estrutura de correlação, permitindo que várias estruturas de covariâncias possam ser implementadas com vistas à obtenção do melhor modelo.
\end{abstract}

Palavras-chave. Modelo Linear Misto, Máxima Verossimilhança, Máxima Verossimilhança Restrita.

\section{Introdução}

Muitas técnicas estatísticas são fundamentadas sob a hipótese de independência entre as observações. De acordo com [2], a falta de independência entre as observações pode resultar em correlação serial, que está presente quando as medidas de uma mesma unidade experimental são tomadas várias vezes ao longo do tempo ou do espaço ou, ainda, quando se tem várias medidas tomadas em uma mesma unidade. Uma vez que, em estudos de medidas repetidas, cada unidade experimental é observada pelo menos duas vezes, pode ocorrer uma dependência entre as respostas e, portanto, grande parte do esforço empregado nas análises deste tipo de dados está relacionada com a modelagem da estrutura das correlações

\footnotetext{
${ }^{1}$ brondino@fc.unesp.br

2 efbchagas@hotmail.com

${ }^{3}$ heu@fc.unesp.br
} 
intra-indivíduos. Dentre as técnicas que permitem esse tipo de modelagem, encontram-se os modelos lineares mistos ([1]).

Desta forma, a possibilidade de modelar as correlações intra-indivíduos encorajou a utilização de um Modelo Linear Misto em um estudo de caso, cujo objetivo foi analisar o impacto de um programa de atividade física nos níveis de glicemia de mulheres na menopausa. Foram consideradas setenta mulheres entre 50 e 79 anos, sedentárias, obesas e sem menstruar por no mínimo doze meses, que foram distribuídas aleatoriamente em grupo treinado $(\mathrm{GT})(\mathrm{n}=35)$ e não treinado $(\mathrm{GnT})(\mathrm{n}=35)$. O GT participou de 20 semanas de um programa de exercícios físicos com três sessões semanais, constituído por atividades de monitoramento e aquecimento (10 minutos); 25 minutos de exercício de flexibilidade e força; 50 minutos de caminhada com intensidade entre 50 a $65 \%$ do $\mathrm{VO}_{2 \max }$; e 5 minutos de volta à calma. $\mathrm{O} \mathrm{GnT}$ foi orientado a manter suas atividades normais. Os valores de glicose plasmática de jejum foram determinados pelo método glicose oxidase, medidos antes e após o período de intervenção do estudo. Para coleta de sangue, todas as pacientes foram orientadas a se apresentarem para o exame em jejum mínimo de 12 horas, e evitar a prática de atividade física vigorosa e a ingestão de álcool nas 24 e 72 horas antecedente à coleta sanguínea, respectivamente. Os procedimentos utilizados nesta pesquisa obedeceram aos Critérios da Ética em Pesquisa com Seres Humanos, conforme resolução 196/96 do Conselho Nacional de Saúde - Brasília/DF, e foram aprovados pelo Comitê de Ética em Pesquisa da Universidade de Marília (UNIMAR), sob o protocolo $n^{\circ} 364$. Para participação no estudo, todas as pacientes assinaram termo de consentimento livre e esclarecido.

\section{O Modelo Linear Misto}

Consideremos $N$ unidades experimentais e $n_{i}$ observações feitas na i-ésima unidade. Sejam $\boldsymbol{y}_{i}$ um vetor $n_{i} \times 1$ de respostas da i-ésima unidade experimental, $\boldsymbol{\beta}$ um vetor $p \times 1$ de parâmetros populacionais desconhecidos (relativo aos efeitos fixos), $\boldsymbol{X}_{i}$ uma matriz conhecida $n_{i} \times p$ de especificação dos efeitos fixos, $\boldsymbol{b}_{i}$ um vetor $k \times 1$ de efeitos individuais desconhecidos (aleatórios), $\boldsymbol{Z}_{i}$ uma matriz conhecida e de especificação de ordem $n_{i} \times k$, dos efeitos aleatórios e $\boldsymbol{e}_{\boldsymbol{i}}$ um vetor $n_{i} \times 1$ de erros aleatórios. Para cada unidade experimental $i$, o modelo linear misto é dado pela equação (1).

$$
\boldsymbol{y}_{i}=\boldsymbol{X}_{i} \boldsymbol{\beta}+\boldsymbol{Z}_{i} \boldsymbol{b}_{i}+\boldsymbol{e}_{i} \quad, i=1,2, \ldots, N
$$

em que os $\boldsymbol{e}_{i}$ apresentam vetor de médias $\mathbf{0}$ e matriz de covariâncias $\boldsymbol{R}_{i}$, de ordem $n_{i} \times n_{i}$, e os vetores $\boldsymbol{b}_{i}$ são independentes entre si e também independentes dos $\boldsymbol{e}_{i}$ e apresentam vetor de médias $\mathbf{0}$ e matriz de covariâncias $\boldsymbol{\Phi}$, de ordem $k \times k$, e as matrizes $\boldsymbol{X}_{i}$ e $\boldsymbol{Z}_{i}$ podem ser formadas tanto por variáveis contínuas quanto binárias.

Se, em adição, $\boldsymbol{e}_{i}$ e $\boldsymbol{b}_{i}$ apresentam distribuição normal, marginalmente os $\boldsymbol{y}_{i}$ são independentes e apresentam distribuição normal com média $\boldsymbol{X}_{i} \boldsymbol{\beta}$ e matriz de covariâncias $\boldsymbol{\Sigma}_{i}=\boldsymbol{Z}_{i} \boldsymbol{\Phi} \boldsymbol{Z}_{i}^{t}+\boldsymbol{R}_{i}$.

As matrizes $\boldsymbol{R}_{i}$ e $\boldsymbol{\Phi}$ são simétricas e positivas definidas e são funções de parâmetros desconhecidos. A escolha das estruturas dessas duas matrizes afetará as estimativas e os erros padrões dos efeitos fixos. Quando $\boldsymbol{R}_{i}=\sigma^{2} \boldsymbol{I}$, onde $\boldsymbol{I}$ é a matriz identidade, as $n_{i}$ observações são independentes condicionadas a $\boldsymbol{b}_{i}$ e $\boldsymbol{\beta}$, e tem-se o denominado modelo de independência condicional. Outras estruturas para essas duas matrizes também podem ser 
utilizadas para acrescentar diferentes estruturas de correlação, tais como a Simetria Composta (SC), a Diagonal (D) e a Não-estruturada (NE), dentre outras ([5]).

$\mathrm{Na}$ forma matricial, o modelo dado em (1) pode ser escrito como em (2).

$$
\boldsymbol{y}=\boldsymbol{X} \boldsymbol{\beta}+\boldsymbol{Z} \boldsymbol{b}+\boldsymbol{e}
$$

em que $N=\sum_{i} n_{i}, \quad \boldsymbol{y}_{N \times 1}=\left[\begin{array}{c}\boldsymbol{y}_{1} \\ \vdots \\ \boldsymbol{y}_{N}\end{array}\right], \quad \boldsymbol{X}=\left[\begin{array}{c}\boldsymbol{X}_{1} \\ \vdots \\ \boldsymbol{X}_{N}\end{array}\right], \quad \boldsymbol{b}=\left[\begin{array}{c}\boldsymbol{b}_{1} \\ \vdots \\ \boldsymbol{b}_{N}\end{array}\right], \quad \boldsymbol{e}=\left[\begin{array}{c}\boldsymbol{e}_{1} \\ \vdots \\ \boldsymbol{e}_{N}\end{array}\right]$;

$\boldsymbol{Z}=\left[\begin{array}{cccc}\boldsymbol{Z}_{1} & \mathbf{0} & \cdots & \mathbf{0} \\ \mathbf{0} & \boldsymbol{Z}_{2} & \cdots & \mathbf{0} \\ \vdots & \vdots & & \vdots \\ \mathbf{0} & \mathbf{0} & \cdots & \boldsymbol{Z}_{N}\end{array}\right] \quad$ e $\quad \boldsymbol{\Sigma}=\left[\begin{array}{cccc}\boldsymbol{\Sigma}_{1} & \mathbf{0} & \cdots & \mathbf{0} \\ \mathbf{0} & \boldsymbol{\Sigma}_{2} & \cdots & \mathbf{0} \\ \vdots & \vdots & & \vdots \\ \mathbf{0} & \mathbf{0} & \cdots & \boldsymbol{\Sigma}_{N}\end{array}\right]$

Uma vez que $\boldsymbol{\Sigma}_{i}=\boldsymbol{Z}_{i} \boldsymbol{\Phi} \boldsymbol{Z}_{i}^{t}+\boldsymbol{R}_{i}$, a matriz de covariâncias de $\boldsymbol{y}$ pode ser escrita como:

$$
\boldsymbol{\Sigma}=\boldsymbol{Z} \boldsymbol{G} \boldsymbol{Z}^{\prime}+\boldsymbol{R}
$$

em que $\boldsymbol{R}=\left[\begin{array}{cccc}\boldsymbol{R}_{1} & \mathbf{0} & \cdots & \mathbf{0} \\ \mathbf{0} & \boldsymbol{R}_{2} & \cdots & \mathbf{0} \\ \vdots & \vdots & & \vdots \\ \mathbf{0} & \mathbf{0} & \cdots & \boldsymbol{R}_{N}\end{array}\right]$ e $\boldsymbol{G}=\left[\begin{array}{cccc}\boldsymbol{\Phi} & \mathbf{0} & \cdots & \mathbf{0} \\ \mathbf{0} & \mathbf{\Phi} & \cdots & \mathbf{0} \\ \vdots & \vdots & & \vdots \\ \mathbf{0} & \mathbf{0} & \cdots & \mathbf{\Phi}\end{array}\right]$

No caso em que $\operatorname{cov}(\boldsymbol{y})=\boldsymbol{\Sigma} \neq \sigma^{2} \boldsymbol{I}$, positiva definida, é conhecida, pode-se usar o Método dos Mínimos Quadrados Generalizados na determinação do BLUE (Best Linear Unbiased Estimator) de $\boldsymbol{\beta}$. Desta forma, $\boldsymbol{\beta}$ é tal que deve minimizar a função objetivo dada em (4). Derivando (4) em relação a $\boldsymbol{\beta}$, temos o sistema de equações normais generalizadas dadas em (5).

$$
\begin{gathered}
(\boldsymbol{y}-\boldsymbol{X} \boldsymbol{\beta})^{t} \boldsymbol{\Sigma}^{-1}(\boldsymbol{y}-\boldsymbol{X} \boldsymbol{\beta}) \\
\boldsymbol{X}^{t} \boldsymbol{\Sigma}^{-1} \boldsymbol{X} \boldsymbol{\beta}=\boldsymbol{X}^{t} \boldsymbol{\Sigma}^{-1} \boldsymbol{y}
\end{gathered}
$$

Uma vez que $\boldsymbol{\Sigma}$ contém os parâmetros de covariância dados pelas matrizes $\boldsymbol{R}$ e $\boldsymbol{G}, \boldsymbol{\beta}$ pode ser expresso em função do vetor de parâmetros $\boldsymbol{\theta}=\left(\boldsymbol{\theta}_{1}, \boldsymbol{\theta}_{2}, \ldots, \boldsymbol{\theta}_{\frac{1}{2} N(N-1)}\right)^{\boldsymbol{t}}$, formado pelas componentes de variância. Dessa forma, a solução do sistema (5) será dada pela equação (6).

$$
\widehat{\boldsymbol{\beta}}=\left(\boldsymbol{X}^{t} \boldsymbol{\Sigma}^{-1}(\boldsymbol{\theta}) \boldsymbol{X}\right)^{-} \boldsymbol{X}^{t} \boldsymbol{\Sigma}^{-1}(\boldsymbol{\theta}) \boldsymbol{y}
$$

em que $\left(\boldsymbol{X}^{t} \boldsymbol{\Sigma}^{-1}(\boldsymbol{\theta}) \boldsymbol{X}\right)^{-}$é uma inversa generalizada qualquer de $\boldsymbol{X}^{t} \boldsymbol{\Sigma}^{-1}(\boldsymbol{\theta}) \boldsymbol{X}$.

No modelo (2), o vetor $\boldsymbol{b}$ é um vetor de variáveis aleatórias. Sob a hipótese de normalidade, o BLUP (Best Linear Unbiased Predictor) de $\boldsymbol{b}$ é um estimador da média condicional $\mathbb{E}(\boldsymbol{b} \mid \boldsymbol{y})$, dado pela expressão (7).

$$
\widehat{\boldsymbol{b}}=\boldsymbol{G} \boldsymbol{Z}^{t} \boldsymbol{\Sigma}^{-1}(\boldsymbol{\theta})(\boldsymbol{y}-\boldsymbol{X} \widehat{\boldsymbol{\beta}})
$$

Na situação em que $\boldsymbol{\Sigma}$ é desconhecida, substitui-se $\boldsymbol{\theta}$ nas expressões (6) e (7) pelo seu estimador $\widehat{\boldsymbol{\theta}}$. Os estimadores resultantes dessa substituição são denominados EBLUE de $\boldsymbol{\beta}$ e EBLUP de $\boldsymbol{b}$, dados por (8) e (9), em que o $E$ nas expressões anteriores denota Empírico.

$$
\begin{aligned}
& \widehat{\boldsymbol{\beta}}=\left(\boldsymbol{X}^{t} \boldsymbol{\Sigma}^{-1}(\widehat{\boldsymbol{\theta}}) \boldsymbol{X}\right)^{-} \boldsymbol{X}^{t} \boldsymbol{\Sigma}^{-1}(\widehat{\boldsymbol{\theta}}) \boldsymbol{y} \\
& \widehat{\boldsymbol{b}}=\boldsymbol{G} \boldsymbol{Z}^{t} \boldsymbol{\Sigma}^{-1}(\widehat{\boldsymbol{\theta}})(\boldsymbol{y}-\boldsymbol{X} \widehat{\boldsymbol{\beta}})
\end{aligned}
$$

Para determinar os estimadores dos parâmetros de covariância contidos em $\widehat{\boldsymbol{\theta}}$, 
usualmente recorre-se aos Métodos de Máxima Verossimilhança (MV) ou Máxima Verossimilhança Restrita (MVR) [3]. No modelo MV, os estimadores dos parâmetros serão dados pela maximização do logaritmo da verossimilhança dado em (10).

$$
l(\boldsymbol{\beta}, \boldsymbol{\theta})=-\frac{N}{2} \ln 2 \pi-\frac{1}{2} \ln |\boldsymbol{\Sigma}(\boldsymbol{\theta})|-\frac{1}{2}(\boldsymbol{y}-\boldsymbol{X} \boldsymbol{\beta})^{t} \boldsymbol{\Sigma}^{-1}(\boldsymbol{\theta})(\boldsymbol{y}-\boldsymbol{X} \boldsymbol{\beta})
$$

Derivando (10) em relação a $\boldsymbol{\beta}$ e igualando a derivada a zero, obtém-se o estimador de $\boldsymbol{\beta}$, $\widehat{\boldsymbol{\beta}}$, dado em (11), o qual coincide com o estimador obtido pela aplicação do Método dos Mínimos Quadrados Generalizados, sob normalidade. Repetindo o processo para a derivada de (10) em relação a $\boldsymbol{\theta}_{q}$, obtém-se as equações para os estimadores dos parâmetros de covariâncias dadas em (12).

$$
\begin{gathered}
\widehat{\boldsymbol{\beta}}=\left(\boldsymbol{X}^{t} \boldsymbol{\Sigma}^{-1} \boldsymbol{X}\right)^{-} \boldsymbol{X}^{t} \boldsymbol{\Sigma}^{-1} \boldsymbol{y} \\
\left.\boldsymbol{y}^{t} \widehat{\boldsymbol{P}} \frac{\partial \boldsymbol{\Sigma}}{\partial \theta_{q}}\right|_{\widehat{\theta}_{q}} \widehat{\boldsymbol{P} \boldsymbol{y}}=\operatorname{tr}\left(\left.\widehat{\boldsymbol{\Sigma}}^{-1} \frac{\partial \boldsymbol{\Sigma}}{\partial \theta_{q}}\right|_{\widehat{\theta}_{q}}\right), q=1,2, \ldots, \frac{N(N+1)}{2}
\end{gathered}
$$

em que $\widehat{\boldsymbol{P}}=\widehat{\boldsymbol{\Sigma}}^{-1}-\widehat{\boldsymbol{\Sigma}}^{-1} \boldsymbol{X}\left(\boldsymbol{X}^{t} \widehat{\boldsymbol{\Sigma}}^{-1} \boldsymbol{X}\right)^{t} \boldsymbol{X}^{t} \widehat{\boldsymbol{\Sigma}}^{-1}$.

O sistema de equações dado em (12) é não linear e só pode ser resolvido numericamente, num processo iterativo. Entre os métodos disponíveis, encontram-se os algoritmos $E M$ (Expectation-Maximization), Newton-Raphson e Fisher Scoring ([5]). Quando $\boldsymbol{\theta}$ não é conhecido, mas uma estimativa $\widehat{\boldsymbol{\theta}}$ está disponível, faz-se $\widehat{\boldsymbol{\Sigma}}=\boldsymbol{\Sigma}(\widehat{\boldsymbol{\theta}})$ nas expressão (10).

A estimativa de MV para $\widehat{\boldsymbol{\theta}}$ é viesada e, como consequência, a matriz de covariâncias de $\widehat{\boldsymbol{\beta}}, \boldsymbol{c o v}(\widehat{\boldsymbol{\beta}})$ também será enviesada, o que interfere na construção dos intervalos de confiança e resultados dos testes de hipóteses. Para reduzir esse viés, pode-se usar o Método MVR ([5]). Esse método consiste em maximizar a verossimilhança de uma transformação ortogonal do tipo $\boldsymbol{y}^{\dagger}=\boldsymbol{U}^{t} \boldsymbol{y}$, em que $\boldsymbol{U}$ é tal que $\mathbb{E}\left(\boldsymbol{y}^{\dagger}\right)=\mathbf{0}$, ou seja, $\boldsymbol{U}^{t} \boldsymbol{X}=\mathbf{0}$, de forma que a verossimilhança obtida a partir dessa transformação não depende dos efeitos fixos $\boldsymbol{\beta}$. Em geral, faz-se $\boldsymbol{U}=\boldsymbol{I}-\boldsymbol{X}\left(\boldsymbol{X}^{t} \boldsymbol{X}\right)^{-1} \boldsymbol{X}^{t}$ ([3]). Sob normalidade, os dados na forma transformada apresentam as seguintes propriedades:

$$
\mathbb{E}\left(\boldsymbol{y}^{\dagger}\right)=\mathbf{0} ; \quad \operatorname{Var}\left(\boldsymbol{y}^{\dagger}\right)=\boldsymbol{U}^{t} \boldsymbol{\Sigma}(\boldsymbol{\theta}) \boldsymbol{U} ; \quad \boldsymbol{y}^{\dagger} \sim N\left(\mathbf{0}, \boldsymbol{U}^{t} \boldsymbol{\Sigma}(\boldsymbol{\theta}) \boldsymbol{U}\right)
$$

O logarítmo da verossimilhança marginal restrita é dado em (14) e a maximização de $l_{R}(\boldsymbol{\theta})$ gera os estimadores de MVR para $\boldsymbol{\theta}$.

$$
\begin{aligned}
l_{R}(\boldsymbol{\theta}) & =-\frac{N-p}{2} \ln 2 \pi-\frac{1}{2} \ln |\boldsymbol{\Sigma}(\boldsymbol{\theta})|-\frac{N-p}{2}(\boldsymbol{y}-\boldsymbol{X} \boldsymbol{\beta})^{t} \boldsymbol{\Sigma}^{-1}(\boldsymbol{\theta})(\boldsymbol{y}-\boldsymbol{X} \boldsymbol{\beta})-\frac{1}{2} \ln \left[\boldsymbol{X}^{t} \boldsymbol{\Sigma}^{-1}(\boldsymbol{\theta}) \boldsymbol{X}\right] \\
\text { em que } p & =\operatorname{rank}(\boldsymbol{U})=N-\operatorname{rank}(\boldsymbol{X}) .
\end{aligned}
$$

Assumindo que as suposições de normalidade são válidas, torna-se necessário avaliar a estrutura da matriz de covariâncias intra-sujeitos, a necessidade da inclusão de efeitos aleatórios e a determinação de uma estrutura de médias que seja apropriada para o modelo. Para avaliar a estrutura da matriz de covariâncias no ajuste de um modelo linear misto com o mesmo número de efeitos fixos, pode-se calcular o valor do log da verossimilhança dos modelos MV ou MVR sob normalidade ([5]). Entre os critérios utilizados para a escolha do melhor modelo, encontram-se os Critérios de Informação de Akaike (AIC) e de Schwarz (BIC), dados pelas expressões (15) e (16). O melhor modelo será aquele que apresenta os menores valores para estas duas medidas. 


$$
\begin{gathered}
A I C=-2 L L(\widehat{\boldsymbol{\theta}})+2 j \\
B I C=-2 L L(\widehat{\boldsymbol{\theta}})+j \cdot \ln (N)
\end{gathered}
$$

em que $L L(\widehat{\boldsymbol{\theta}})$ é o máximo da função log-verossimilhança e $j$ é o número de parâmetros a serem estimados no modelo.

Outro método para escolha do modelo é o Teste da Razão de Verossimilhanças, que é indicado para comparar dois modelos aninhados, com mesmos efeitos fixos. A hipótese nula estabelece que o modelo com menos parâmetros representa bem o conjunto de dados. Se a hipótese nula é rejeitada, então o modelo mais completo é mais adequado. A estatística de teste $d$, dada em (17), apresenta Distribuição Qui-quadrado com $v$ graus de liberdade, onde $v$ é dado pela diferença no número de parâmetros dos dois modelos ([5]).

$$
d=-2 L L \text { do modelo completo }+2 L L \text { do modelo reduzido }
$$

em que $L L$ denota o máximo do logaritmo neperiano da verossimilhança.

De acordo com [4], quando modelos são ajustados pelo Método MVR, as comparações de modelos através dos testes da razão de verossimilhanças restritas devem ser limitadas aos modelos que podem ser encaixados, de acordo com os parâmetros da matriz de covariâncias. Como consequência, modelos que apresentam diferentes matrizes de planejamento não podem ser comparados com base na Máxima Verossimilhança Restrita. Nesta situação, a escolha da estrutura do modelo deve ser feita através da comparação das medidas de qualidade de ajuste AIC e BIC.

\section{Aplicação a um estudo de caso}

$\mathrm{Na}$ especificação do modelo, foram utilizadas as variáveis dicotômicas $G R$ e $P$, para identificar o grupo e o período, respectivamente, sendo $G R=0$ para o grupo treinado, $G R=1$ para o grupo não treinado, $P=0$ para o período pré-intervenção e $P=1$ para o período pós-intervenção. Os valores de glicemia foram medidos em $\mathrm{mg} / \mathrm{dl}$.

Com o objetivo de avaliar os efeitos fixos e aleatórios a serem considerados, a primeira etapa consistiu da simulação de 22 modelos, cujas estruturas são apresentadas na Tabela $1 \mathrm{e}$ aos quais foram aplicados tanto o método MV quanto o método MVR. Nesta etapa, as estruturas das matrizes $\boldsymbol{R}$ e $\boldsymbol{G}$, quando o caso, foram escolhidas como não estruturadas (NE).

Dentre os modelos constantes na Tabela 1, que só apresenta resultados para o método MVR, os menores valores para AIC e BIC foram obtidos para as estruturas 1,10 e 11 . Podese observar que, numa comparação entre os três modelos, os maiores valores de AIC e BIC foram observados para o primeiro, indicando que as duas estruturas com menos parâmetros mostraram-se superiores. Os resultados ainda indicam que os modelos 10 e 11 apresentam valores iguais para AIC e BIC. Desta forma, o critério de desempate entre os dois deu-se em função dos menores valores para os parâmetros de covariâncias estimados para $\boldsymbol{R}$ e $\boldsymbol{G}$, tendo em vista a interferência destes nos intervalos de confiança obtidos para o vetor $\boldsymbol{\beta}$. As covariâncias apresentadas na Tabela 2 indicam que, com exceção da variância estimada para o período pós intervenção, todas as outras medidas apresentadas para o modelo 10 são menores. Observa-se que a variabilidade entre os indivíduos sofreu um aumento importante (de 218,61 no modelo 10 para 327,92 no modelo 11), indicando que a estrutura do modelo 10 é a mais adequada. 
Definidos os parâmetros fixos e aleatórios a serem incluídos no modelo, o próximo passo consistiu na escolha da estrutura da matriz de covariâncias $\boldsymbol{R}$, que melhor se adaptava ao problema. As medidas de qualidade de ajuste para o modelo 10 e diferentes estruturas da matriz $\boldsymbol{R}$ são apresentadas na tabela 3. O número de parâmetros das estruturas CS, AR(1) e D é o mesmo e, portanto, a escolha dentre as três deu-se de acordo com os menores valores observados para AIC, BIC e -2LL, sendo que a estrutura D mostrou-se a mais adequada. O Teste da Razão de Verossimilhanças foi utilizado para comparar os modelos que poderiam ser aninhados de acordo com os parâmetros da matriz de covariâncias, conforme Tabela 4.

Tabela 1: Estrutura dos modelos simulados e medidas de qualidade de ajuste.

\begin{tabular}{|c|c|c|c|c|c|}
\hline Modelo & Efeitos Fixos & Efeitos Aleatórios & AIC & BIC & -2LL \\
\hline $\mathbf{1}$ & I, GR, P, INT & I, P & 1225,96 & 1252,17 & 1207,96 \\
\hline $\mathbf{2}$ & I, GR, P & I, P & 1243,89 & 1270,17 & 1225,89 \\
\hline $\mathbf{3}$ & I, GR, P & I & 1237,89 & 1255,41 & 1225,89 \\
\hline $\mathbf{4}$ & I, GR, P & P & 1250,60 & 1268,16 & 1238,60 \\
\hline $\mathbf{5}$ & I, GR & I, P & 1250,60 & 1268,16 & 1238,60 \\
\hline $\mathbf{6}$ & I, GR & I & 1246,60 & 1258,31 & 1238,60 \\
\hline $\mathbf{7}$ & I, GR & P & 1246,60 & 1258,31 & 1238,60 \\
\hline $\mathbf{8}$ & I, P & I, P & 1243,54 & 1261,10 & 1231,54 \\
\hline $\mathbf{9}$ & I, P & P & 1239,54 & 1251,25 & 1231,54 \\
\hline $\mathbf{1 0}$ & I, GR, P, INT & I & 1215,96 & 1227,61 & 1207,96 \\
\hline $\mathbf{1 1}$ & I, GR, P, INT & P & 1215,96 & 1227,61 & 1207,96 \\
\hline
\end{tabular}

Notações - GR: grupo; P: período; INT: interação; I: intercepto.

Tabela 2: Parâmetros de covariância estimados para os modelos 10 e 11.

\begin{tabular}{|c|c|c|}
\hline \multirow{2}{*}{ Parâmetro } & Modelo 10 & Modelo 11 \\
\cline { 2 - 3 } & Estimativa & Estimativa \\
\hline Variância no pré-intervenção & 124,11 & 342,73 \\
\hline Covariância entre os períodos pré e pós & $-23,66$ & 194,95 \\
\hline Variância no pós-intervenção & 313,61 & 203,81 \\
\hline Variância entre indivíduos & 218,61 & 327,92 \\
\hline
\end{tabular}

Tabela 3: Medidas de qualidade de ajuste para diferentes estruturas para a matriz $\boldsymbol{R}$.

\begin{tabular}{|c|c|c|c|c|}
\hline Estrutura de $\boldsymbol{R}$ & AIC & BIC & -2LL & Número de parâmetros \\
\hline NE & 1215,96 & 1227,61 & 1207,96 & 8 \\
\hline SC & 1218,04 & 1226,78 & 1212,04 & 7 \\
\hline AR(1) & 1218,04 & 1226,78 & 1212,04 & 7 \\
\hline D & 1213,96 & 1222,69 & 1207,96 & 7 \\
\hline I & 1216,04 & 1221,87 & 1212,04 & 6 \\
\hline
\end{tabular}

Notações - NE: não estruturada; SC: simetria composta; AR: auto-regressivo; D: diagonal; I: identidade.

Tabela 4: Teste da Razão de Verossimilhanças.

\begin{tabular}{|c|c|c|c|c|c|c|c|}
\hline & SC vs. NE & AR(1) vs. NE & I vs. NE & D vs. NE & I vs. SC & I vs. AR(1) & I vs. D \\
\hline$d$ & 4,09 & 4,09 & 4,09 & $\approx 0$ & $\approx 0$ & $\approx 0$ & 4,09 \\
\hline p-valor & 0,04 & 0,04 & 0,13 & $>0,99$ & $>0,99$ & $>0,99$ & 0,04 \\
\hline Melhor estrutura & NE & NE & I & D & I & I & D \\
\hline
\end{tabular}


Considerando os resultados apresentados nas Tabelas 1 a 4, as variáveis $G R, P$ e o fator de interação $G R \times P$ foram incluídos como efeitos fixos, o intercepto foi incluído como efeito aleatório e a estrutura de $\boldsymbol{R}$ escolhida foi a Diagonal.

$\mathrm{Na}$ sequência, os parâmetros para os efeitos fixos foram obtidos a partir de (10), gerando a equação de estimação para os níveis de glicemia médios dada em (18). A significância dos parâmetros foi analisada através do teste t-Student, indicando que a interação $G R \times P$ foi significativa a um nível $\alpha=5 \%(p-$ value $<0,0001)$, ou seja, os dois grupos comportaram-se de maneiras distintas ao longo do tempo.

$$
\text { Glicemia }=96,714-1,486 \times G R-1,086 \times P+19,571 \times G R \times P
$$

\section{Conclusões}

Este trabalho utilizou-se de um Modelo Linear Misto para analisar o efeito de atividade física nos níveis de glicemia de mulheres obesas na menopausa. A justificativa dessa abordagem encontra-se no fato de que, em experimentos que envolvem medidas repetidas, $o$ esperado é que as respostas medidas em uma unidade experimental não sejam independentes. Além disso, esta abordagem permite que várias estruturas de matrizes de covariâncias possam ser testadas, possibilitando o ajuste de um modelo com menos parâmetros.

Os resultados obtidos indicaram que a interação $G R \times P$ mostrou-se significativa a um nível $\propto=5 \%$, ou seja, os dois grupos comportaram-se de maneiras distintas ao longo do tempo. A metodologia utilizada também permitiu a construção de uma equação para estimar o nível médio de glicemia, a partir da qual pode-se concluir que, após a intervenção, o nível de glicemia para o grupo das ativas $(G R=0)$ sofreu uma redução média de aproximadamente $1,086 \mathrm{mg} / \mathrm{dl}$, enquanto para o grupo das inativas $(G R=1)$, o mesmo sofreu um aumento médio de aproximadamente $17,0 \mathrm{mg} / \mathrm{dl}$.

\section{Referências}

[1] N. M Laird and J. H., Ware, Random-Effects Models for Longitudinal Data, Biometrics, vol. 38, 963-974, (1982).

[2] A. C. Rencher and G. B. Schaalje, Linear Models in Statistics - 2nd ed., John Wiley \& Sons, (2008).

[3] J. M. Singer, J. S. Nobre e F. M. M. Rocha, Análise de Dados Longitudinais - versão parcial preliminar, (2012), disponível em:

<http://www.ime.usp.br/ jmsinger/Textos/Singer\&Nobre\&Rocha2012mar.pdf> Acesso em 08.abril.2014.

[4] M. C. Stein, Testes da Razão de Verossimilhanças em Modelos Lineares Mistos, Dissertação de Mestrado em Estatística, Departamento de Estatística, Instituto de Ciências Exatas, Universidade Federal de Minas Gerais, (2008).

[5] N. H. Timm, Applied Multivariate Analysis, Springer, (2002). 